\title{
Nonlocal Form of Quantum Off-Shell Kinetic Equation*
}

\author{
Yu.B. Ivanov ${ }^{1, \dagger}$ and D.N. Voskresensky ${ }^{1, \dagger}$ \\ ${ }^{1}$ Gesellschaft für Schwerionenforschung $m b H$, \\ Planckstr. 1, 64291 Darmstadt, Germany
}

\begin{abstract}
A new nonlocal form of the off-shell kinetic equation is derived. While being equivalent to the Kadanoff-Baym and Botermans-Malfliet formulations in the range of formal applicability, it has certain advantages beyond this range. It possesses more accurate conservation laws for Noether quantities than those in the Botermans-Malfliet formulation. At the same time the nonlocal form, similarly to the BotermansMalfliet one, allows application of the test-particle method for its numerical solution, which makes it practical for simulations of heavy-ion collisions. The physical meaning of the time-space nonlocality is clarified.
\end{abstract}

November 4, 2018

\section{INTRODUCTION}

The work of S.T. Belyaev and G.I. Budker, who demonstrated the Lorentz invariance of the relativistic distribution function and derived relativistic Fokker-Planck kinetic equation [1], stands in the line of achievements of the kinetic theory. This work entered many textbooks and found numerous applications in various fields. Presently the relativistic transport concepts are a conventional tool to analyze the dynamics of dense and highly excited matter produced in relativistic heavy-ion collisions.

A great progress was also achieved in microscopic foundation of the kinetic theory. The appropriate frame for description of non-equilibrium processes within the real-time formalism of quantum-field theory was developed by Schwinger, Kadanoff, Baym and Keldysh [2, 3, 4]. The formalism allows extensions of the quantum kinetic picture beyond conventional

\footnotetext{
* Dedicated to S.T. Belyaev on the occasion of his 85th birthday. 'Y.Ivanov@gsi.de; RRC "Kurchatov Institute", Kurchatov sq. 1, Moscow 123182, Russia 市D.Voskresensky@gsi.de; Moscow Institute for Physics and Engineering, Kashirskoe sh. 31, Moscow 115409, Russia
} 
approximations (like the quasiparticle one). This is caused by the quest for dynamical treatment of broad resonances as well as stable particles which acquire a considerable mass width because of collisional broadening. The above mentioned applications request for development of approximate self-consistent schemes possessing conservation laws being at least approximately satisfied [5, 6, 7, 8, 9, 10, 11]. Based on these schemes, numerical transport methods for treatment of the off-shell dynamics have been developed [12, 13, 14].

Two slightly different forms of the Kadanoff-Baym equations expanded up to first-order space-time gradients are now used: the proper Kadanoff-Baym (KB) form, as it follows right after the gradient expansion of exact KB equations [3], and the Botermans-Malfliet (BM) one, as it follows after a modification of a Poisson-bracket term in the KB equation [15]. Both the $\mathrm{KB}$ and $\mathrm{BM}$ forms coincide in the first-order gradient approximation but differ in higher orders. Both forms have their advantages and disadvantages [10]. The KB form possesses exact conservation laws for the Noether current and the energy-momentum [9, 10], however, it does not allow the efficient test-particle method to be applied to its numeric solution. The BM form is very suitable for the test-particle method [12, 13, 14] but only approximately conserves the Noether current and the energy-momentum.

In this paper we would like to put forward a new, space-time nonlocal form of the quantum kinetic equation, which combines favorable features of the above mentioned $\mathrm{KB}$ and $\mathrm{BM}$ forms. In sect. [1] we start with brief review of properties of the KB and BM forms of the off-shell kinetic schemes. Technical details are deferred to the Appendix A. In sect. III the new nonlocal form of the quantum kinetic equation is derived and physical meaning of the nonlocality is clarified. Possible applications in numerical transport schemes are discussed.

\section{KADANOFF-BAYM AND BOTERMANS-MALFLIET KINETICS}

In this section we summarize the formulation of the off-shell kinetic equations in the two different forms: in the $\mathrm{KB}$ form and in the $\mathrm{BM}$ form. We assume the reader is familiar with the real-time formulation of non-equilibrium many-body theory and use of the contour matrix notation, detailed in Appendix $\mathrm{A}$.

Starting point of all considerations is the set of Kadanoff-Baym equations which express 
the space-time changes of the Wigner transformed ${ }^{1}$ correlation function $\mathrm{i}^{-+}(X, p)$ in terms of the real-time contour convolution of the self-energy $\Sigma$ with the Green function $G$. We give the kinetic equation in compact notation (cf. Eq. (A4))

$$
v_{\mu} \partial_{X}^{\mu} \mathrm{i} G^{-+}(X, p)=[\Sigma \otimes G-G \otimes \Sigma]_{X, p}^{-+} \quad \text { with } \quad v^{\mu}=\frac{\partial}{\partial p_{\mu}} G_{0}^{-1}(p),
$$

where $G_{0}^{-1}(p)$ is the Fourier transform of the inverse free Green function

$$
G_{0}^{-1}(p)= \begin{cases}p^{2}-m^{2} & \text { for relativistic bosons } \\ p_{0}-\boldsymbol{p}^{2} /(2 m) & \text { for non-rel. fermions or bosons. }\end{cases}
$$

For a complete definition, Eq. (1) has to be supplemented with further equations, e.g., for the retarded Green function together with the retarded relations (A6). If a system under consideration is only slightly spatially inhomogeneous and slowly evolving in time, a good approximation is provided by an expansion up to first order in space-time gradients. Then the main problem to arrive at a proper kinetic equation consists in accurately disentangling a rather complicated r.h.s. of Eq. (11).

\section{A. $\Phi$-derivable approximations}

In actual calculations one often uses approximations or truncation schemes to the exact non-equilibrium theory, where conservation laws (such as charge and energy-momentum conservations) and thermodynamic consistency of the transport theory are not evident. It was shown [5, 6, 7] that there exists a class of self-consistent approximations, called $\Phi$ derivable approximations, which are conserving at the expectation value level, i.e. they provide true Noether currents and a conserved energy-momentum tensor, and at the same time thermodynamically consistent. In these schemes the self-energies are self-consistently generated from a functional $\Phi[G]$ through the following variational procedure [7]

$$
-\mathrm{i} \Sigma_{i k}(X, p)=\mp \frac{\delta \mathrm{i} \Phi[G]}{\delta \mathrm{i} G^{k i}(X, p)} \times\left\{\begin{array}{ll}
2 & \text { for real fields } \\
1 & \text { for complex fields }
\end{array}, \quad i, k \in\{-+\} .\right.
$$

The functional $\Phi[G]$ specifies a truncation scheme. It consists of a set of properly chosen closed two-particle irreducible diagrams, where lines denote the self-consistent propagators

\footnotetext{
${ }^{1}$ The space-time variable is $X \equiv X^{\mu} \equiv(t, \mathbf{x})$, and the Fourier transformed variable is $p \equiv p^{\mu}$.
} 
$G$, while vertices are bare. The functional variation with respect to $G$ diagrammatically implies an opening of a propagator line of $\Phi$.

\section{B. Physical notation}

It is useful to eliminate the imaginary factors inherent in the standard Green function formulation and introduce quantities which are real and possess clear physical meaning. Thus instead of Green functions $G^{i j}(X, p)$ and self-energies $\Sigma^{i j}(X, p)$ with $i, j \in\{-+\}$ in the Wigner representation we use the kinetic notation of Refs. [7, 10]. We define the generalized distribution functions $F$ and $\widetilde{F}$ in the 8-dimensional phase space,

$$
F(X, p)=(\mp) \mathrm{i} G^{-+}(X, p), \quad \widetilde{F}(X, p)=\mathrm{i} G^{+-}(X, p) .
$$

Here and below the upper sign corresponds to fermions, while the lower sign, to bosons,

$$
A(X, p) \equiv-2 \operatorname{Im} G^{R}(X, p)=\widetilde{F} \pm F
$$

is the spectral function, and $G^{R}$ is the retarded propagator. The spectral function satisfies the sum rule

$$
\begin{aligned}
\int_{0}^{\infty} \frac{\mathrm{d} p_{0}}{2 \pi} A(X, p) & =1 \quad \text { for nonrelativ. particles, } \\
\int_{-\infty}^{\infty} \frac{\mathrm{d} p_{0}}{2 \pi} p_{0} A(X, p) & =1 \quad \text { for relativ. bosons }
\end{aligned}
$$

which follows from the canonical equal-time (anti)commutation relations for (fermionic) bosonic field operators. The gain and loss rates of the collision term are defined as

$$
\Gamma^{\mathrm{in}}(X, p)=\mp \mathrm{i} \Sigma^{-+}(X, p), \quad \Gamma^{\mathrm{out}}(X, p)=\mathrm{i} \Sigma^{+-}(X, p)
$$

with the damping width

$$
\Gamma(X, p) \equiv-2 \operatorname{Im} \Sigma^{R}(X, p)=\Gamma^{\text {out }}(X, p) \pm \Gamma^{\text {in }}(X, p)
$$

where $\Sigma^{R}$ is the retarded self-energy.

In terms of above kinetic notation, the gradient-expanded Kadanoff-Baym equations are reduced to equations for real quantities: for the real and imaginary parts of the retarded Green function $G^{R}$, and for the phase-space occupation $F$. Note that the number of 
equations for $G^{i j}$ Green functions is four, which twice exceeds the number of unknown functions $\left(F\right.$ and $\left.G^{R}\right)$. Before the gradient expansion all equations were completely consistent. However, after the gradient expansion their interrelation is no longer obvious. Necessary interrelations have been derived in Refs. [7, 10].

The equations for the retarded propagator in the first-order gradient approximation can be immediately solved with the result [3, 15]

$$
G^{R}=\frac{1}{M(X, p)+\mathrm{i} \Gamma(X, p) / 2} \Rightarrow\left\{\begin{aligned}
A(X, p) & =\frac{\Gamma(X, p)}{M^{2}(X, p)+\Gamma^{2}(X, p) / 4} \\
\operatorname{Re} G^{R}(X, p) & =\frac{M(X, p)}{M^{2}(X, p)+\Gamma^{2}(X, p) / 4}
\end{aligned}\right.
$$

with the "mass" function

$$
M(X, p)=G_{0}^{-1}(p)-\operatorname{Re} \Sigma^{R}(X, p) .
$$

Note that the algebraic solution (9) is valid within the first-order gradient approximation, i.e. only the terms $O\left(\partial_{X}^{2}\right)$ are omitted.

\section{Kadanoff-Baym form of kinetic equation}

In terms of above notation, the KB kinetic equation for $F$ in the first-order gradient approximation takes the form, see [7] for details,

$$
\mathcal{D} F(X, p)-\left\{\Gamma^{\mathrm{in}}, \operatorname{Re} G^{R}\right\}=C(X, p) .
$$

We refer this as the kinetic equation in the KB-choice ${ }^{2}$. Here $\{\ldots, \ldots\}$ denotes the fourdimensional Poisson bracket (A10). The differential drift operator is defined as

$$
\mathcal{D}=\left(v_{\mu}-\frac{\partial \operatorname{Re} \Sigma^{R}}{\partial p^{\mu}}\right) \partial_{X}^{\mu}+\frac{\partial \operatorname{Re} \Sigma^{R}}{\partial X^{\mu}} \frac{\partial}{\partial p_{\mu}},
$$

note that $\mathcal{D} \psi=\{M, \psi\}$ for arbitrary function $\psi$. The collision term is defined as

$$
C(X, p)=\Gamma^{\text {in }}(X, p) \widetilde{F}(X, p)-\Gamma^{\text {out }}(X, p) F(X, p) .
$$

\footnotetext{
${ }^{2}$ If the system consists of different particle species, there is a set of coupled kinetic equations corresponding to each species.
} 
In terms of a functional $\Phi$ the explicit form of the collision term is

$$
C(X, p)=\frac{\delta \mathrm{i} \Phi}{\delta \widetilde{F}(X, p)} \widetilde{F}(X, p)-\frac{\delta \mathrm{i} \Phi}{\delta F(X, p)} F(X, p),
$$

cf. Eq. (3)

Within the consistent gradient expansion $C \propto \partial_{X}$. If the diagrams for the self-energy contain internal vertices, it gives rise to non-local effects in the collision term

$$
C=C_{\mathrm{loc}}+C_{\mathrm{mem}}
$$

Here $C_{\text {loc }}$ is a local part of $C$, where all these nonlocalities are disregarded, and $C_{\text {mem }} \propto$ $\partial_{X}$ is the nonlocal part expanded up to first gradient terms. Following [7] we call these effects the memory effects. Explicit form of the $C_{\text {mem }}$ depends on the specific system under consideration.

For the sake of clarity, below we do few simplifications. We do not consider the memory effects, implying that $C=C^{\text {loc }}$. Besides, we confine ourselves to the case void of derivative coupling. Also we do not explicitly introduce the particle-specie label to avoid overcomplication of equations.

The local part of the collision term is charge and energy-momentum conserving by itself

$$
\operatorname{Tr} \int \frac{\mathrm{d}^{4} p}{(2 \pi)^{4}}\left(\begin{array}{l}
e \\
p^{\mu}
\end{array}\right) C^{\mathrm{loc}}=0
$$

Here $e$ denotes a charge (e.g., the baryon number), while Tr implies the sum over all possible internal degrees of freedom (like spin, isospin, etc.) and over possible particle species.

The true Noether current of the charge $e$,

$$
j^{\mu}(X)=e \operatorname{Tr} \int \frac{\mathrm{d}^{4} p}{(2 \pi)^{4}} v^{\mu} F(X, p),
$$

is exactly conserved

$$
\partial_{\mu} j^{\mu}(X)=0
$$

which follows right from the operator expression for this quantity, cf. Ref. [7].

On the other hand, the integration of the KB equation (11) permits us to derive another, "effective KB current"

$$
j_{\text {KB-eff }}^{\mu}(X)=e \operatorname{Tr} \int \frac{\mathrm{d}^{4} p}{(2 \pi)^{4}}\left[\left(v_{\mu}-\frac{\partial \operatorname{Re} \Sigma^{R}}{\partial p^{\mu}}\right) F-\operatorname{Re} G^{R} \frac{\partial \Gamma^{\mathrm{in}}}{\partial p_{\mu}}\right]
$$


which is exactly conserved. The equivalence of (17) and (19) and thus the exact conservation of the Noether current (17) follows from the corresponding invariance of the $\Phi$ functional, cf. Eq. (6.9) in [6], which results in the consistency relation

$$
e \operatorname{Tr} \int \frac{\mathrm{d}^{4} p}{(2 \pi)^{4}}\left\{-\partial_{\mu}\left[\frac{\partial \operatorname{Re} \Sigma^{R}}{\partial p^{\mu}} F+\operatorname{Re} G^{R} \frac{\partial \Gamma^{\mathrm{in}}}{\partial p_{\mu}}\right]+C\right\}=0 .
$$

Similar situation takes place for the energy-momentum conservation. Here we also rely on $\Phi$-derivable approximations which provide the corresponding exact conservation.

The conserving feature is especially important for devising numerical simulation codes based on this kinetic equation. Indeed, if a test-particle method is used, one should be sure that the number of test particles is exactly conserved rather than approximately. In the test-particle method the distribution function is represented by an ensemble of test particles as follows

$$
F(X, p) \sim \sum_{i} \delta^{(3)}\left(\mathbf{x}-\mathbf{x}_{i}(t)\right) \delta^{(4)}\left(p-p_{i}(t)\right)
$$

where the $i$-sum runs over test particles. Then the $\mathcal{D} F$ term in Eq. (11) just corresponds to the classical motion of these test particles subjected to forces inferred from $\operatorname{Re} \Sigma^{R}$, while the collision term $C$ gives stochastic change of test-particle's momenta, when their trajectories "cross". For a direct application of this method, however, there is a particular problem with the KB kinetic equation. The additional term, i.e. the Poisson-bracket term $\left\{\Gamma^{\mathrm{in}}, \operatorname{Re} G^{R}\right\}$, spoils this simplistic picture, since derivatives acting on the distribution function $F$ appear here only indirectly and thus cannot be included in the collisionless propagation of test particles. This problem, of course, does not prevent a direct solution of the KB kinetic equation. For instance, one can apply well developed lattice methods, which are, however, much more complicated and time-consuming as compared to the test particle approach.

\section{Botermans-Malfliet form of kinetic equation}

As can be seen from Eqs. (44), (8) and (13), the gain rate $\Gamma^{\text {in }}$ differs from $F \Gamma / A$ only by corrections of the first order in the gradients

$$
\Gamma^{\text {in }}=\Gamma F / A+C / A=\Gamma F / A+O\left(\partial_{X}\right),
$$

since $C \sim O\left(\partial_{X}\right)$. This fact permits us to neglect the correction $O\left(\partial_{X}\right)$, as in the kinetic equation it leads to terms of already second-order in the gradients. Upon substitution 
$\Gamma^{\text {in }}=\Gamma F / A$, proposed by Botermans and Malfliet [15], one arrives at the following form of the kinetic equation, see [7] for details,

$$
\mathcal{D} F(X, p)-\left\{\Gamma \frac{F}{A}, \operatorname{Re} G^{R}\right\}=C(X, p),
$$

which is still equivalent to the KB form within the first-order gradient approximation (all terms $\propto O\left(\partial_{X}^{2}\right)$ are now omitted). We call the so obtained Eq. (23) the kinetic equation in $B M$-choice. All KB-choice properties of Eq. (23) within a $\Phi$-derivable approximation also transcribe to BM-choice through the substitution $\Gamma^{\text {in }}=\Gamma F / A$ in the consistency relation Eq. (201).

The BM equation exactly conserves the following "effective BM current"

$$
\begin{aligned}
j_{\mathrm{BM}-\mathrm{eff}}^{\mu}(X) & =e \operatorname{Tr} \int \frac{\mathrm{d}^{4} p}{(2 \pi)^{4}}\left[v^{\mu} F(X, p)+\operatorname{Re} \Sigma^{R} \frac{\partial F}{\partial p_{\mu}}-\operatorname{Re} G^{R} \frac{\partial(\Gamma F / A)}{\partial p_{\mu}}\right] \\
& =e \operatorname{Tr} \int \frac{\mathrm{d}^{4} p}{(2 \pi)^{4}} \frac{\Gamma}{2} B^{\mu} F
\end{aligned}
$$

where

$$
B^{\mu} \equiv\left(B_{0}, \boldsymbol{B}\right)=A\left[\left(v^{\mu}-\frac{\partial \operatorname{Re} \Sigma^{R}}{\partial p_{\mu}}\right)-\frac{M}{\Gamma} \frac{\partial \Gamma}{\partial p_{\mu}}\right]
$$

is the flow spectral function introduced in [7]. The 0-component of the flow spectral function $B^{\mu}$ obeys the same sum-rule as the spectral function $A$, cf. [16]. The effective current (24) differs from the true Noether current $j^{\mu}$ of Eq. (17) and the effective KB current (19) in terms of the order of $O\left(\partial_{X}\right)$, provided a $\Phi$-derivable approximation is used for self-energies. Thus with the BM choice the conservation laws of the Noether current (17) and the energymomentum tensor are only approximately fulfilled.

The effective BM-current (24) was used by S. Leupold [14] as a basis for the construction of a test-particle ansatz for numerical solution of the nonrelativistic BM kinetic equation. To automatically fulfill the effective current conservation, the test-particle ansatz is introduced for the combination

$$
\frac{1}{2} \Gamma B_{0} F(X, p) \sim \sum_{i} \delta^{(3)}\left(\mathbf{x}-\mathbf{x}_{i}(t)\right) \delta^{(4)}\left(p-p_{i}(t)\right)
$$

rather than for the distribution function itself. Note that the energy $p_{i}^{0}(t)$ of the test particle is an independent coordinate, not restricted by a mass-shell condition. W. Cassing and S. Juchem [13] used this test-particle ansatz in the relativistic case. 
The BM kinetic equation (23) together with ansatz (26) for the distribution function result in the following set of equations for evolution of parameters of the test particles between collisions

$$
\begin{aligned}
\dot{\mathbf{x}}_{i} & =\frac{1}{v_{0}-\partial_{E_{i}} \operatorname{Re} \Sigma^{R}-(M / \Gamma) \partial_{E_{i}} \Gamma}\left(\mathbf{v}_{i}+\nabla_{p_{i}} \operatorname{Re} \Sigma^{R}+(M / \Gamma) \nabla_{p_{i}} \Gamma\right), \\
\dot{\mathbf{p}}_{i} & =\frac{1}{v_{0}-\partial_{E_{i}} \operatorname{Re} \Sigma^{R}-(M / \Gamma) \partial_{E_{i}} \Gamma}\left(\nabla_{x} \operatorname{Re} \Sigma^{R}+(M / \Gamma) \nabla_{x_{i}} \Gamma\right), \\
\dot{E}_{i} & =\frac{1}{v_{0}-\partial_{E_{i}} \operatorname{Re} \Sigma^{R}-(M / \Gamma) \partial_{E_{i}} \Gamma}\left(\partial_{t} \operatorname{Re} \Sigma^{R}+(M / \Gamma) \partial_{t} \Gamma\right) .
\end{aligned}
$$

These equations of motion, in particular, give the time evolution of the mass term $M$, of a test particle [13, 14]

$$
\frac{\mathrm{d} M_{i}}{\mathrm{~d} t}=\frac{M_{i}}{\Gamma_{i}} \frac{\mathrm{d} \Gamma_{i}}{\mathrm{~d} t}
$$

the origin of which can be traced back to the additional term $\left\{\Gamma F / A, \operatorname{Re} G^{R}\right\}$ in the BM equation (23). Here $M_{i}(t)=M\left[t, \mathbf{x}_{i}(t) ; E_{i}(t), \mathbf{p}_{i}(t)\right]$ measures an "off-shellness" of the test particle, and $\Gamma_{i}(t)=\Gamma\left[t, \mathbf{x}_{i}(t) ; E_{i}(t), \mathbf{p}_{i}(t)\right]$. Equation of motion (30) yields $M_{i}=\alpha_{i} \Gamma_{i}$, where $\alpha_{i}$ do not depend on time, and implies that once the width drops in time the particles are driven towards the on-shell mass, i.e. to $M=0$. This clarifies the meaning of the additional term $\left\{\Gamma F / A, \operatorname{Re} G^{R}\right\}$ in the off-shell $\mathrm{BM}$ transport: it provides the time evolution of the off-shellness.

The problem of not exactly conserving Noether charges within the BM kinetics can be mitigated in certain cases. For instance, if we start from initial conditions defined for particles in vacuum (where $\Sigma=0$ and hence both charges coincide) and end the evolution also in a very dilute state, the Noether charge turns out to be conserved in the end of the evolution. However, this is not the case with heavy-ion collisions, where we start with two cold nuclei.

\section{NONLOCAL FORM OF OFF-SHELL KINETIC EQUATION}

\section{A. Nonlocal kinetic equation and conservation laws}

Let us rewrite the KB kinetic equation (11) in the following non-local (NL) form

$$
\mathcal{D} F(X, p)-\left\{\Gamma \frac{F}{A}, \operatorname{Re} G^{R}\right\}=C^{\mathrm{NL}}
$$




$$
\equiv\left(1+\left\{\frac{1}{A}, \operatorname{Re} G^{R}\right\}\right) C\left(X^{\mu}-\frac{1}{A} \frac{\partial \operatorname{Re} G^{R}}{\partial p_{\mu}}, p^{\mu}+\frac{1}{A} \frac{\partial \operatorname{Re} G^{R}}{\partial X_{\mu}}\right) .
$$

Eq. (31) is the key equation of our work. The collision term $C^{\mathrm{NL}}$ is expressed here in terms of shifted variables. Note that the specific 8-phase-space memory in $C^{\mathrm{NL}}$ should not be confused with the memory effects resulting from internal structure of self-energy diagrams with more than two vertices. In order to distinguish between these two effects we call the non-locality in Eq. (31), as delays (positive or negative) in 8-phase-space rather than the memory.

To verify that the NL kinetic equation Eq. (31) is equivalent to the KB one, including second-order gradient terms, let us expand the collision term $C^{\mathrm{NL}}$ in small quantities

$$
\delta X^{\mu}=(\delta \tau, \delta \mathbf{x})=\frac{1}{A} \frac{\partial \operatorname{Re} G^{R}}{\partial p_{\mu}}, \quad \delta p^{\mu}=\frac{1}{A} \frac{\partial \operatorname{Re} G^{R}}{\partial X_{\mu}} .
$$

Then

$$
\begin{aligned}
C^{\mathrm{NL}} & =\left(1+\left\{\frac{1}{A}, \operatorname{Re} G^{R}\right\}\right)\left(C+\frac{1}{A}\left\{C, \operatorname{Re} G^{R}\right\}\right)+O\left(\partial_{X}^{3}\right) \\
& =C+\left\{C / A, \operatorname{Re} G^{R}\right\}+O\left(\partial_{X}^{3}\right) .
\end{aligned}
$$

Here we have taken into account that $C \propto O\left(\partial_{X}\right)$. The resulting Poisson bracket $\left\{C / A, \operatorname{Re} G^{R}\right\}$ combines with the Poisson bracket on the l.h.s., cf. Eq. (22), resulting in $\left\{\Gamma^{\text {in }}, \operatorname{Re} G^{R}\right\}$, as it stands in the KB kinetic equation (11). Thus, indeed, Eq. (31) coincides with the KB equation (11) within the approximation including second-order terms in space-time gradients. The roughening of this approximation by neglecting the second-order terms (i.e., if one puts $C^{\mathrm{NL}}=C$ ), returns us to the BM equation.

The consistency condition related to Eq. (31) can be rewritten as

$$
e \operatorname{Tr} \int \frac{\mathrm{d}^{4} p}{(2 \pi)^{4}}\left\{-\partial_{\mu}\left[\frac{\partial \operatorname{Re} \Sigma^{R}}{\partial p^{\mu}} F+\operatorname{Re} G^{R} \frac{\partial(\Gamma F / A)}{\partial p_{\mu}}\right]+C^{\mathrm{NL}}+O\left(\partial_{X}^{3}\right)\right\}=0,
$$

cf. Eq. (20). Thus, the precision of conservations of the Noether baryon number and the energy-momentum in the NL equation is one order in space-time gradients higher than that in the BM equation. Indeed, the effective BM current differs from the Noether one in terms of the first-order gradients whereas the effective current that follows from the NL kinetic equation differs only in second-order gradients. The latter takes place because the NL equation is equivalent to the $\mathrm{KB}$ equation including $O\left(\partial_{X}^{2}\right)$ terms. Let us recollect that Noether currents are exactly conserved for the KB choice [9]. 
Within the range of formal applicability, the NL equation is equivalent to the KB and BM forms discussed above. However, in practical calculations one frequently needs to apply the kinetic approach beyond the scope of its applicability. For instance, such a problem arises when one describes the initial stage of heavy ion collisions. We do not know how good the quantum kinetic equations are beyond the region of their formal applicability, but we certainly wish to respect conservation laws. From this point of view the KB form is certainly preferable. As the conservations are exact, we can still use the gradient approximation, relying on a minor role of this rather short initial stage of heavy ion collisions in the total evolution of a system. Exact conservation laws allow us to keep control of numerical codes. However, as we have mentioned, the efficient test-particle method is not applicable for solution of the KB equation. At the same time, for the BM kinetic equation the testparticle method is already available [13, 14], for the price that it deals with the approximately conserved Noether current.

When the collision term $C$ is large, the neglect of the $C / A$ term in the r.h.s. of the relation (22) is a bad approximation. Please, recollect that this approximation is in the basis of the BM approximation. Therefore, the nonlocal form of the kinetic equation which we present here is certainly preferable. Conservations of the Noether current and energy-momentum are more accurate here than in the BM form, since they are closer to exact conservations of the $\mathrm{KB}$ form. ${ }^{3}$ At the same time the test-particle method is applicable to the NL equation. In the NL case the set of equations for evolution of parameters of the test particles between collisions is the same as in the BM case, see Eqs. (27)-(29). The only difference with the BM case is that collisions of test particles occur with certain time (and space) delay (or advance) as compared with the instant of their closest approach to each other. Because of this circumstance the effective BM current (24) is no longer locally conserved. Indeed, integrating $C^{\mathrm{NL}}$ over 4-momentum and taking into account relation (33), we arrive at

$$
\begin{aligned}
\operatorname{Tr} \int \frac{\mathrm{d}^{4} p}{(2 \pi)^{4}} C^{\mathrm{NL}} & =\operatorname{Tr} \int \frac{\mathrm{d}^{4} p}{(2 \pi)^{4}}\left\{C / A, \operatorname{Re} G^{R}\right\}+O\left(\partial_{X}^{3}\right) \\
& =\partial_{\mu}\left(-\operatorname{Tr} \int \frac{\mathrm{d}^{4} p}{(2 \pi)^{4}} \frac{C}{A} \frac{\partial \operatorname{Re} G^{R}}{\partial p_{\mu}}\right)+O\left(\partial_{X}^{3}\right) .
\end{aligned}
$$

Precisely this contribution makes the KB effective current (19) from the corresponding BM

\footnotetext{
${ }^{3}$ Deriving (31), we only added higher (than second order) space-time derivatives to equation in KB choice (11).
} 
current (24). This nonconservation does not prevent us from application of the test-particle method. Since collisions are nonlocal in the time-space, we need only global conservation of the BM effective current, which in fact takes place

$$
\operatorname{Tr} \int \mathrm{d}^{4} X \frac{\mathrm{d}^{4} p}{(2 \pi)^{4}} C^{\mathrm{NL}}=\int \mathrm{d}^{4} X \partial_{\mu}\left(-\operatorname{Tr} \int \frac{\mathrm{d}^{4} p}{(2 \pi)^{4}} \frac{C}{A} \frac{\partial \operatorname{Re} G^{R}}{\partial p_{\mu}}\right)+O\left(\partial_{X}^{2}\right)=O\left(\partial_{X}^{2}\right) .
$$

Now this conservation is approximate, $O\left(\partial_{X}^{2}\right)$ terms are dropped. Nevertheless, this precision is better than that resulting from the formal accuracy of the kinetic equations, where already $O\left(\partial_{X}\right)$ terms are dropped in the approximately conserving quantities.

Actually we could modify the NL equation in such a way that it exactly fulfills global conservation of the effective charge, which may be important for the test-particle method. Indeed, we recognize that to the first-order gradients the pre-factor $J=\left(1+\left\{\frac{1}{A}, \operatorname{Re} G^{R}\right\}\right)$ in Eq. (31) is the Jacobian $J$ of the transformation from $\mathrm{d}^{4} p \mathrm{~d}^{4} X$ to the $\mathrm{d}^{4} \widetilde{p} \mathrm{~d} \widetilde{X}$ integration expressed in terms of shifted variables $\widetilde{p}^{\mu}=p^{\mu}+\delta p^{\mu}$ and $\widetilde{X}^{\mu}=X^{\mu}-\delta X^{\mu}$. By adding terms $\delta J$ of higher (than $O\left(\partial_{X}\right)$ ) orders to $J$, we do not violate the nature of the NL approximation. Then $\delta J$ can be tuned in such a way that $J+\delta J$ becomes the exact Jacobian of the transformation and hence the BM effective current turns out to be exactly globally conserved:

$$
\operatorname{Tr} \int \mathrm{d}^{4} X \frac{\mathrm{d}^{4} p}{(2 \pi)^{4}} \frac{J+\delta J}{J} C^{\mathrm{NL}}=\operatorname{Tr} \int \mathrm{d}^{4} \widetilde{X} \frac{\mathrm{d}^{4} \widetilde{p}}{(2 \pi)^{4}} C(\widetilde{X}, \widetilde{p})=0 .
$$

However the explicit expression for $\delta J \propto O\left(\partial_{X}^{2}\right)$ is very cumbersome. In order to keep the formalism transparent, we avoid this extra complication.

\section{B. Time delays and causality}

The NL kinetic equation (31) contains the delayed collision term and the BM particle drift term including the drag and the back flow contributions. The extra Poisson-bracket term $\left\{C / A, \operatorname{Re} G^{R}\right\}$ which in the $\mathrm{KB}$ equation had a poorly defined physical meaning is now hidden in the 8-phase-space delays of the collision term thus acquiring a new physical interpretation. The space-time part of the retardation/advance (32) is expressed as

$$
\delta X^{\mu}=\frac{1}{2} B^{\mu}-\frac{1}{\Gamma}\left(v^{\mu}-\frac{\partial \operatorname{Re} \Sigma^{R}}{\partial p_{\mu}}\right)
$$

where we used Eq. (9) and the definition of the flow spectral function (25)). There are two contributions to the $\delta X^{\mu}$. The first term is proportional to the same $B^{\mu}$ function 
that determines the trajectories of the test particles between collisions, see Eqs. (27)-(29). The second term $\left(v^{\mu}-\partial \operatorname{Re} \Sigma^{R} / \partial p_{\mu}\right) / \Gamma$ relates to collisions: $1 / \Gamma \sim 1 /(u \sigma \rho)$ in the gas approximation, where $u$ is the particle velocity, $\rho$ is the density of the medium and $\sigma$ is the cross-section.

Note that the time delay in the collision term has no definite sign (see discussion in the next subsection). Therefore, one could naively suppose that NL kinetic equation violates causality. Here the following comment is in order. The initial Dyson equations for non-equilibrium Green functions are causal. The apparent problem appears already at the moment when one chooses $t=\left(t_{1}+t_{2}\right) / 2$ and $\Delta t=t_{2}-t_{1}$ as independent variables (where $t_{2}>t_{1}$ due to causality) to perform then the Wigner transformation. Although approximations have not yet been done, in new variables the Green function $G^{R}(t, \Delta t)$ contains an information from the future, see Fig. 19 of Ref. [17]. The problem would completely disappear, if one had used $t_{1}$ and $\Delta t=t_{2}-t_{1}$ as independent variables. Thus, working in $t=\left(t_{1}+t_{2}\right) / 2$ and $\Delta t=t_{2}-t_{1}$ variables we incorporate some information from the future. Otherwise our results would be incompatible with those obtained from the original Dyson equations. However, this fact does not contradict causality, since the Dyson equations are causal and approximations have not yet been done. The necessity to include the information from the future is clearly seen, e.g., from analysis of the $\Phi$-diagrams containing more than two vertices. The vertices depend on different time variables. These dependencies reveal themselves in the above mentioned memory effects in the gradient expanded kinetic equations.

The apparent acausality disappears after the first-order gradient expansion, and it does not manifest in the $\mathrm{KB}$ and $\mathrm{BM}$ forms of the kinetic equations since all variables there depend locally on the single time $t$. However via corresponding Poisson bracketed terms these equations contain an information on the mentioned non-local effects, which were originally present. Constructing (31) we re-grouped some of these terms hiding them in the collision term at the price that the apparent problem again appeared. However, the NL kinetic equation can be read from the right to the left in such a way that the collision term in future is determined by the drift term at present. This way the NL equation is again completely causal.

As we will see in the next section the new NL form of the kinetic equation is convenient to discuss the so called negative time delays which have been experimentally observed in semi- 
conductors, see [18] and refs. therein. The quantum kinetic delay effects reflect fundamental physical limitations which follow from basic uncertainty principles for the time-energy and the coordinate-momentum. The finite duration (positive or negative) implies that the final state of the system is not entirely determined until the process is completed. The wave-like nature of excitations indicates that the interactions between waves are interference phenomena which have finite duration on a scale of at least one period of the interacting waves. Collisions cannot be considered to be completed at times shorter than these periods. This may result in that a collision in progress can be reversed at some conditions. Quantum nature of collisions is reflected in the formalism of quantum kinetics contrary to the Boltzmann kinetics dealing with free particle propagation between instant collisions.

\section{Physical meaning of time delays and advances}

In order to consider the variable shifts in the collision term in more detail, let us present the time delay as a sum of two quantities

$$
\delta \tau=\delta \tau_{\mathrm{drift}}+\delta \tau_{\mathrm{col}} \equiv \frac{B_{0}}{2}-\frac{Z^{-1}}{\Gamma},
$$

where $Z=\left(v_{0}-\partial \operatorname{Re} \Sigma^{R} / \partial p^{0}\right)^{-1}$ is the normalization factor which usually appears in description of quasiparticle effects. In contrast to the quasiparticle case, here $p_{0}$ and $\boldsymbol{p}$ are independent variables (not connected by any dispersion relation). The $B_{0}$ function can be formally expressed as

$$
B_{0}=2 \frac{\partial \delta}{\partial p_{0}}, \quad \tan \delta=-\frac{\Gamma}{2 M} .
$$

The latter ratio is a measure of proximity of a virtual particle with given $p_{0}$ and $\boldsymbol{p}$ to the mass-shell, see Eq. (30) above.

The value $\delta \tau_{\text {drift }}=B_{0} / 2$ has the meaning of the drift delay time, $\delta \tau_{\text {col }}=-Z^{-1} / \Gamma$, of the collision delay time, and $\delta \tau$, of the effective total delay time. In the virial limit these time delays coincide with those studied in [19, 20]. In particular, for quasiparticles $Z>0$ and $M \rightarrow 0$, and therefore we obtain $B_{0}=Z A=2 \pi Z \delta(M)>0$ and hence $\delta \tau_{\text {drift }}$ is positive delay, and $\delta \tau_{\text {col }}$ is negative delay at arbitrary densities. To further clarify the meaning of these delays let us consider several examples.

- $\pi N \Delta$ system 
If we consider propagation of a resonance, e.g., $\Delta$ isobar, in the virial limit of very small densities, $\delta$ has the meaning of the $\pi N$ phase shift. Thus in the virial limit, $B_{0}$ of the resonance relates to the energy variation of the scattering phase shift $\left(\delta_{33}^{\pi N}\right)$ of the channel coupled to the resonance, cf. [16]. Due to the Galilean invariance the vacuum Green function and self-energy depend only on the energy in the $\pi N$ center-of-mass frame and $B_{0}$ is recovered from the known dependence $\delta_{33}^{\pi N}\left(E_{\text {c.m. }}\right)$. As it follows from this dependence, $B_{0}>0$ and hence $\delta \tau_{\text {drift }}>0$ (delay). There also appears a negative delay $\delta \tau_{\text {col }}<0$ (for $Z>0$ ), since namely during the $Z_{\Delta}^{-1} / \Gamma_{\Delta}$ time the $\Delta$ resonance state forms the intermediate state of the $\pi N$ pair (causing an advance), compare with Ref. [20]. The $\delta \tau$ value in Eq. (39) has no definite sign. Its sign depends on which time is dominant, $\delta \tau_{\text {drift }}$ or $\delta \tau_{\text {col }}$. If we consider the pion propagation, $B_{0}=B_{0}^{\pi}$ and in the virial limit $\delta$ has the meaning of the in-medium $\Delta-$ nucleon hole phase shift. For the nucleon, $B_{0}^{N}$ is related to $\delta_{\pi \Delta}$.

\section{- Wigner resonances}

For a Wigner nonrelativistic resonance, when $\operatorname{Re} \Sigma^{R}$ and $\Gamma$ are independent of $p$, we have $Z=1$. Then the value $\delta \tau$ in Eq. (39) becomes

$$
\delta \tau=-\frac{M^{2}-\Gamma^{2} / 4}{\Gamma\left(M^{2}+\Gamma^{2} / 4\right)},
$$

which precisely coincides with the result Ref. [20]. As it follows from (41), in the vicinity of the resonance $(M \rightarrow 0) \delta \tau$ is positive (delay) and outside the resonance region $\left(M^{2} \gg \Gamma^{2}\right) \delta \tau$ is negative (advance). If the interaction is attractive, the particle spends a shorter time in the interaction zone than that would be, if it moved with unchanged asymptotic velocity. This case corresponds to a delay. If the interaction is repulsive, the particle vise versa spends a longer time in the interaction zone, that corresponds to a negative delay. Thus, close to the Wigner resonance we may speak about an effective attraction and outside the resonance region, about an effective repulsion.

\section{- Soft photon radiation}

Let us consider another example [21] of the photon radiation in the propagation of a source charge (say a proton) in neutral matter (e.g. the neutron one). The source particle looses the memory about collisions which have happened long ago (after a 
time $\tau \gg\left|\delta \tau_{\text {col }}\right|$ has past). The advance in the collision time can be understood in terms of multiple scatterings. The latter act coherently for $\tau \lesssim\left|\delta \tau_{\text {col }}\right| \sim 1 / \Gamma$. Indeed, for energies of the radiating quantum corresponding to the wavelength $\lambda_{\gamma}=2 \pi / \omega_{\gamma} \gtrsim$ $\left|\delta \tau_{\text {col }}\right|$ the source particle simultaneously feels all rescatterings which it had and also will have on the time scale $\left|\delta \tau_{\text {col }}\right|$. This results in the well known Landau-PomeranchukMigdal effect [22] experimentally proven at the Stanford Linear Accelerator Center [23]. For large photon energies, $\lambda_{\gamma}$ is small and radiation occurs on a single center (neutron), whereas soft radiation occurs coherently on many centers. Thus appearance of the time advances $\delta \tau_{\text {col }}$ in this example is associated with the multiple rescattering effects.

In the above examples $Z$ factors were assumed to be positive. However, $Z$ can be negative in some energy-momentum regions far from the mass-shell, cf. [24]. Change of the sign of $Z$ is reflected in the respective sign change in $\delta \tau_{\mathrm{col}}$.

Moreover, interactions lead to the change in the generalized group velocity

$$
\boldsymbol{u}_{\text {group }}=Z\left(\boldsymbol{v}+\frac{\partial \operatorname{Re} \Sigma^{R}}{\partial \boldsymbol{p}}\right)
$$

that reflects in the time-space delays. To illustrate it let us consider an example of the pion propagation in the isospin-symmetric nuclear matter. For the sake of simplicity, we consider the pion propagation in a region close to the $\Delta$-resonance and assume that the pion interacts with the nucleons only through the $\pi N \Delta$-coupling $f_{\pi N \Delta}$. Then the real part of the pion self-energy becomes [24]

$$
\operatorname{Re} \Sigma_{\pi} \simeq \frac{\alpha_{0} f_{\pi N \Delta}^{2} \boldsymbol{k}^{2} \rho_{N} \omega_{\Delta}\left[\omega^{2}-\omega_{\Delta}^{2}\right]}{\left(\omega^{2}-\omega_{\Delta}^{2}\right)^{2}+\Gamma^{2} \omega_{\Delta}^{2}}
$$

Here $\omega_{\Delta}=m_{\Delta}-m_{N}, \rho_{N}$ is the nucleon matter density, $\alpha_{0}=$ const $\sim 1$. From here we can find $Z_{\pi}$-factor. For $\omega \rightarrow \omega_{\Delta}$ we obtain

$$
Z_{\pi} \rightarrow\left[2 \omega_{\Delta}-\frac{2 \alpha_{0} f_{\pi N \Delta}^{2} \boldsymbol{k}^{2} \rho_{N}}{\Gamma_{\Delta}^{2}}\right]^{-1}, \quad \boldsymbol{u}_{\text {group }} \rightarrow 2 \boldsymbol{k} Z
$$

resulting in an increase of the group velocity in the resonance region. Here it is worthwhile to note that for light in a dielectric medium there appears increase in the group velocity for packets with resonant frequencies [25]. 


\section{Space and energy-momentum shifts}

As follows from Eq. (32) the shift of the space variables is

$$
\delta \boldsymbol{x}=\frac{1}{A} \frac{\partial \operatorname{Re} G^{R}}{\partial \boldsymbol{p}}=\delta \boldsymbol{x}_{\mathrm{drift}}+\delta \boldsymbol{x}_{\mathrm{col}}=\frac{1}{2} \boldsymbol{B}+\boldsymbol{u}_{\mathrm{group}} \delta \tau_{\mathrm{col}} .
$$

We can further express the spatial shift (45) in terms of time delays (39) and velocity $\dot{\mathbf{x}}$ of a test particle on its trajectory, ${ }^{4}$ cf. Eq. (27),

$$
\delta \boldsymbol{x}=\dot{\mathbf{x}} \delta \tau_{\text {drift }}+\boldsymbol{u}_{\text {group }} \delta \tau_{\text {col }} .
$$

Eq. (46) demonstrates that to a delayed collision the test particle moves along its trajectory. Therefore, the drift time delay $\delta \tau_{\text {drift }}$ unambiguously results in a definite space shift $\dot{\mathbf{x}} \delta \tau_{\text {drift }}$. The collision itself is associated with an additional time delay $\delta \tau_{\text {col }}$, which implies that the collision is not instant, as it is treated in the kinetic equation, but requires certain time for complete decoupling from intermediate states (e.g., the pion spends some time in the intermediate $\Delta$-nucleon-hole state, a soft photon requires certain time to be formed in multiple collisions of the proton with neutrons). Therefore, this additional delay gives rise to an additional shift $\boldsymbol{u}_{\text {group }} \delta \tau_{\text {col }}$ of the particle with respect to its "collisionless" trajectory (27).

Since during time-delay interval $\delta \tau$ a particle propagates in the mean field $\operatorname{Re} \Sigma^{R}$, it also changes its energy and momentum. The energy-momentum shift (32) can be represented in a similar form

$$
\delta p^{\mu}=\dot{p}^{\mu} \delta \tau_{\text {drift }}-Z \frac{\partial \operatorname{Re} \Sigma^{R}}{\partial X_{\mu}} \delta \tau_{\text {col }},
$$

where $\dot{p}^{\mu}=(\dot{E}, \dot{\mathbf{p}})$ is the rate of change of the 4-momentum of a test particle on its trajectory, cf. Eqs. (28) and (29). We again distinctly see two contributions to $\delta p^{\mu}$. The first one is associated with the energy-momentum change during the path of the particle to a delayed collision. The second one is related to an intrinsic nonlocal character of the collision itself. Thus, we see that all shifts in space and energy-momentum variables are consistent with time delays discussed above.

The energy shift can be expressed in the alternative form

$$
\delta p_{0}=\frac{M^{2}-\Gamma^{2} / 4}{M^{2}+\Gamma^{2} / 4}\left(\frac{\partial_{t} \operatorname{Re} \Sigma^{R}}{\Gamma}\right)-\operatorname{Re} G^{R} \partial_{t} \Gamma
$$

\footnotetext{
${ }^{4}$ Here for briefness we omit the unnecessary subscript $i$ of the test particle.
} 
where we used Eq. (9). In the case of the Wigner resonance, the first term on the r.h.s. of this relation is $-\delta \tau \cdot \partial_{\tau} \operatorname{Re} \Sigma^{R}$. Thus, the energy-shift scale is $\sim\left|\operatorname{Re} \Sigma^{R} \cdot \delta \tau\right| / \tau_{\mathrm{ch}}$, where $\tau_{\mathrm{ch}}$ is the characteristic time of the self-energy variation. In the virial limit $\operatorname{Re} \Sigma^{R}=4 \pi \rho \operatorname{Re} F(0)$, where $F(0)$ is the forward scattering amplitude in the energetic normalization, $\rho$ is the density of the medium. Then we obtain

$$
\delta p_{0} \sim-4 \pi \operatorname{Re} F(0) \delta \tau \cdot \partial_{t} \rho,
$$

thus relating the value $\tau_{\mathrm{ch}}$ to the variation of the density. In the kinetic region the $\tau_{\mathrm{ch}}$ scale is the collisional time, $\tau_{\mathrm{ch}} \sim\left|\delta \tau_{\mathrm{col}}\right|$, and hence the correction $\delta p_{0}$ can be not small. In the region of the validity of the hydrodynamics the density varies on a much longer scale than the collisional one, and hence the correction $\delta p_{0}$ is small but still does not vanish. When the global equilibrium is reached this correction disappears.

Since the time delay occurs in all the quantities entering the collision term, and the value of the $\delta \tau$ delay is finite even in the case of global equilibrium (e.g., see Eq. (41)), the thermodynamic quantities, such as the pressure, energy, etc., also acquire corrections proportional to this time delay.

At the general level, the relation of thermodynamic properties to the time delays can be understood in terms of ergodicity. The system spends certain time in a certain phase-space region, which is proportional to the density of states in that region. Change in this time corresponds to the change in the density of states and thereby to the change in thermodynamic properties, cf. [20]. We have generalized this statement to the unified 8-phase-space.

Another important feature of the kinetic description is approaching to thermal equilibrium during the evolution of a closed system. In terms of transport theory it means that the H-theorem takes place for transport equations, which implies construction of the entropy related to the kinetic equation. These problems have been considered both for the BM equation and for the KB equation [7, 8, 9, 10]. Since the NL form of the quantum kinetic equation (31) is equivalent to the KB equation including second-order terms in space-time gradients, the NL entropy differs from the KB entropy only in the second-order gradient terms. This reduces the problem of the H-theorem for the new NL kinetic equation to the already solved problem for the KB equation [10]. 


\section{SUMMARY}

We have considered properties (advantages and disadvantages) of available formulations of the off-shell kinetic equation, i.e. those in the Kadanoff-Baym and Botermans-Malfliet forms, as well as a new nonlocal form which we derived in this paper. Within the range of formal applicability, all these forms are equivalent to each other. Under this applicability range we mean states of a system which are close to equilibrium, where the space-time gradient expansion is strictly applicable. In particular, it implies that conservation laws of Noether currents and energy-momentum are satisfied at least to the extent of validity of the dynamics, i.e. up to zero-order gradients. Note that these conservation laws are even exactly fulfilled for the Kadanoff-Baym form [9]. However, these equations are frequently applied beyond the scope of their applicability, e.g., when the collision term is large. Such a situation takes place, e.g., in description of the initial stage of heavy-ion collisions. In such situations we certainly wish to respect conservation laws which allow us to keep control of numerical codes. From this point of view the KB form is certainly preferable. As the conservations are exact, we can still use the gradient approximation, relying on a minor role of this rather short initial stage of heavy ion collisions in the total evolution of a system. However, the efficient test-particle method is not applicable for solution of the Kadanoff-Baym equation.

The nonlocal kinetic equation differs from the Kadanoff-Baym one only in third-order gradient terms, while the Botermans-Malfliet form, in the second order. Due to this the nonlocal form of the kinetic equation is certainly preferable as compared with the Botermans-Malfliet one, since it more accurately conserves the Noether currents and the energy-momentum. Deviations from the Noether quantities appear only in the second gradient order, as compared with first order for the Botermans-Malfliet equation. In addition, the nonlocal form allows application of the test-particle method very similarly to that has been done for the Botermans-Malfliet form [13, 14]. Presently, there are numeric schemes dealing with delayed (advanced) collisions of the test particles [20, 26]. Thus, the nonlocal form of the quantum kinetic equation is a reasonable compromise between the Kadanoff-Baym and BotermansMalfliet equations, which is practical like the Botermans-Malfliet form and at the same time reasonably preserves conservations of Noether quantities.

Physical meaning of delays and advances in 8-dimensional phase space appearing in the nonlocal form of the kinetic equation is clarified. It is demonstrated that in addition to 
the practical utility the nonlocal form provides us with clear physical interpretation of various terms in the Kadanoff-Baym equation and also allows us to consider delicate physical phenomena, which are beyond the scope of the Botermans-Malfliet approximation.

\section{Acknowledgments}

We are grateful to J. Knoll for valuable remarks and constructive criticism. Also we acknowledge E.E. Kolomeitsev for fruitful discussions. This work was supported by the Deutsche Forschungsgemeinschaft (DFG project 436 RUS 113/558/0-3), the Russian Foundation for Basic Research (RFBR grant 06-02-04001 NNIO_a), and Russian Federal Agency for Science and Innovations (grant NSh-3004.2008.2).

\section{Appendix A: MATRIX NOTATION}

In calculations that apply the Wigner transformations, it is necessary to decompose the full contour into its two branches-the time-ordered and anti-time-ordered branches. One then has to distinguish between the physical space-time coordinates $x, \ldots$ and the corresponding contour coordinates $x^{\mathcal{C}}$ which for a given $x$ take two values $x^{-}=\left(x_{\mu}^{-}\right)$and $x^{+}=\left(x_{\mu}^{+}\right)(\mu \in\{0,1,2,3\})$ on the two branches of the contour (see figure 1$)$.

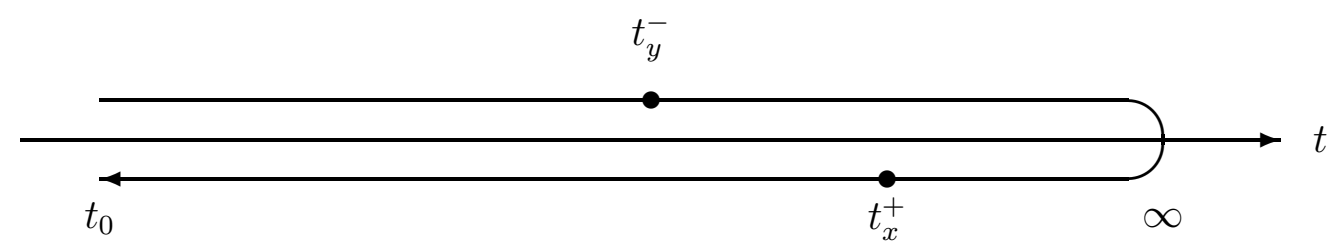

Figure 1: Closed real-time contour with two external points $x, y$ on the contour.

Closed real-time contour integrations can then be decomposed as

$$
\int_{\mathcal{C}} \mathrm{d} x \ldots=\int_{t_{0}}^{\infty} \mathrm{d} x \ldots+\int_{\infty}^{t_{0}} \mathrm{~d} x \ldots=\int_{t_{0}}^{\infty} \mathrm{d} x \ldots-\int_{t_{0}}^{\infty} \mathrm{d} x \ldots,
$$

where only the time limits are explicitly given. The extra minus sign of the anti-time-ordered branch can conveniently be formulated by a $\{-+\}$ "metric" with the metric tensor in $\{-+\}$ indices

$$
\left(\sigma^{i j}\right)=\left(\sigma_{i j}\right)=\left(\begin{array}{cc}
1 & 0 \\
0 & -1
\end{array}\right)
$$


which provides a proper matrix algebra for multi-point functions on the contour with "co"and "contra"-contour values. Thus, for any two-point function $F$, the contour values are defined as

$$
\begin{aligned}
F^{i j}(x, y) & :=F\left(x^{i}, y^{j}\right), \quad i, j \in\{-,+\}, \quad \text { with } \\
F_{i}{ }^{j}(x, y) & :=\sigma_{i k} F^{k j}(x, y), \quad F^{i}{ }_{j}(x, y):=F^{i k}(x, y) \sigma_{k j} \\
F_{i j}(x, y) & :=\sigma_{i k} \sigma_{j l} F^{k l}(x, y), \quad \sigma_{i}^{k}=\delta_{i k}
\end{aligned}
$$

on the different branches of the contour. Here summation over repeated indices is implied. Then contour folding of contour two-point functions, e.g., in Dyson equations, simply becomes

$$
\begin{aligned}
H\left(x^{i}, y^{k}\right) & =H^{i k}(x, y)=[F \otimes G]^{i k} \\
& \equiv \int_{\mathcal{C}} \mathrm{d} z F\left(x^{i}, z\right) G\left(z, y^{k}\right)=\int \mathrm{d} z F^{i}{ }_{j}(x, z) G^{j k}(z, y)
\end{aligned}
$$

in the matrix notation.

Due to the change of operator ordering, genuine multi-point functions are, in general, discontinuous, when ever two contour coordinates become identical. In particular, two-point functions like $\mathrm{i} F(x, y)=\left\langle\mathcal{T}_{\mathcal{C}} \widehat{A}(x) \widehat{B}(y)\right\rangle$ become $^{5}$

$$
\mathrm{i} F(x, y)=\left(\begin{array}{cc}
\mathrm{i} F^{--}(x, y) & \mathrm{i} F^{-+}(x, y) \\
\mathrm{i} F^{+-}(x, y) & \mathrm{i} F^{++}(x, y)
\end{array}\right)=\left(\begin{array}{cc}
\langle\mathcal{T} \widehat{A}(x) \widehat{B}(y)\rangle & \mp\langle\widehat{B}(y) \widehat{A}(x)\rangle \\
\langle\widehat{A}(x) \widehat{B}(y)\rangle & \left\langle\mathcal{T}^{-1} \widehat{A}(x) \widehat{B}(y)\right\rangle
\end{array}\right)
$$

where $\mathcal{T}$ and $\mathcal{T}^{-1}$ are the usual time and anti-time ordering operators. Since there are altogether only two possible orderings of the two operators, in fact given by the Wightman functions $F^{-+}$and $F^{+-}$, which are both continuous, not all four components of $F$ are independent. Eq. (A5) implies the following relations between nonequilibrium and usual retarded and advanced functions

$$
\begin{aligned}
F^{R}(x, y) & =F^{--}(x, y)-F^{-+}(x, y)=F^{+-}(x, y)-F^{++}(x, y) \\
& :=\Theta\left(x_{0}-y_{0}\right)\left(F^{+-}(x, y)-F^{-+}(x, y)\right), \\
F^{A}(x, y) & =F^{--}(x, y)-F^{+-}(x, y)=F^{-+}(x, y)-F^{++}(x, y) \\
& :=-\Theta\left(y_{0}-x_{0}\right)\left(F^{+-}(x, y)-F^{-+}(x, y)\right),
\end{aligned}
$$

\footnotetext{
${ }^{5}$ Frequently used alternative notation is $F^{<}=F^{-+}$and $F^{>}=F^{+-}$.
} 
where $\Theta\left(x_{0}-y_{0}\right)$ is the step function of the time difference. The rules for the co-contour functions $F_{--}$etc. follow from Eq. (A3).

For slightly inhomogeneous and slowly evolving systems, the degrees of freedom can be subdivided into rapid and slow ones. Any kinetic approximation is essentially based on this assumption. Then for any two-point function $F^{i j}(x, y)$, one separates the variable $\xi=\left(t_{1}-t_{2}, \boldsymbol{r}_{\mathbf{1}}-\boldsymbol{r}_{\mathbf{2}}\right)$, which relates to rapid and short-ranged microscopic processes, and the variable $X=\frac{1}{2}\left(t_{1}+t_{2}, \boldsymbol{r}_{\mathbf{1}}+\boldsymbol{r}_{\mathbf{2}}\right)$, which refers to slow and long-ranged collective motions. The Wigner transformation, i.e. the Fourier transformation in four-space difference $\xi=x-y$ to four-momentum $p$

$$
F^{i j}(X ; p)=\int \mathrm{d} \xi e^{\mathrm{i} p \xi} F^{i j}(X+\xi / 2, X-\xi / 2), \quad i, j \in\{-+\}
$$

where $X=(t, \mathbf{x})$, leads to the corresponding Wigner densities in four-phase-space. The gradient expansion converts the Wigner transformation of any convolution of two-point functions into a product of the corresponding Wigner functions plus higher order gradient terms

$$
\begin{aligned}
\int \mathrm{d} \xi e^{\mathrm{i} p \xi}\left(\int \mathrm{d} z f(x, z) \varphi(z, y)\right) & =\left(\exp \left[\frac{\mathrm{i} \hbar}{2}\left(\partial_{p} \partial_{X^{\prime}}-\partial_{X} \partial_{p^{\prime}}\right)\right] f(X, p) \varphi\left(X^{\prime}, p^{\prime}\right)\right)_{p^{\prime}=p, X^{\prime}=X}(\mathrm{~A} 8) \\
& \simeq f(X, p) \varphi(X, p)+\frac{\mathrm{i} \hbar}{2}\{f(X, p), \varphi(X, p)\}, \quad \text { (A9) }
\end{aligned}
$$

where the first order terms are given by Poisson brackets

$$
\{f(X, p), \varphi(X, p)\}=\frac{\partial f}{\partial p^{\mu}} \frac{\partial \varphi}{\partial X_{\mu}}-\frac{\partial f}{\partial X^{\mu}} \frac{\partial \varphi}{\partial p_{\mu}}
$$

here in covariant notation. We would like to stress that the smallness of the $\hbar \partial_{X} \cdot \partial_{p}$ comes solely from the smallness of space-time gradients $\partial_{X}$, while momentum derivatives $\partial_{p}$ are not assumed to be small. This point is sometimes incorrectly treated in the literature.

[1] S.T. Belyaev and G.I. Budker, Dokl. AN SSSR 107, 807 (1965).

[2] J. Schwinger, J. Math. Phys. 2, 407 (1961).

[3] L.P. Kadanoff and G. Baym, Quantum Statistical Mechanics, Benjamin, 1962.

[4] L.P. Keldysh, ZhETF 47, 1515 (1964) [ Sov. Phys. JETP 20, 1018 (1965)].

[5] G. Baym, Phys. Rev. 127, 1391 (1962).

[6] Yu.B. Ivanov, J. Knoll, and D.N. Voskresensky, Nucl. Phys. A 657, 413 (1999). 
[7] Yu.B. Ivanov, J. Knoll and D.N. Voskresensky, Nucl. Phys. A 672, 313 (2000).

[8] Yu.B. Ivanov, J. Knoll, H. van Hees and D. N. Voskresensky, Yad. Fiz. 64, 711 (2001).

[9] J. Knoll, Yu.B. Ivanov, and D.N. Voskresensky, Ann. Phys. (NY) 293 , 126 (2001).

[10] Yu.B. Ivanov, J. Knoll and D.N. Voskresensky, Yad. Fiz. 66, 1950 (2003) [Phys. Atom. Nucl. 66, $1902(2003)]$.

[11] J. Knoll, F. Riek, Yu.B. Ivanov and D.N. Voskresensky, J. Phys. Conf. Ser. 35, 357 (2006).

[12] M. Effenberger, U. Mosel, Phys. Rev. C 60, 51901 (1999).

[13] W. Cassing and S. Juchem, Nucl. Phys. A 665, 377 (2000); Nucl. Phys. A 672, 417 (2000).

[14] S. Leupold, Nucl. Phys. A 672, 475 (2000).

[15] W. Botermans and R. Malfliet, Phys. Rep. 198, 115 (1990).

[16] W. Weinhold, B.L. Friman and W. Nörenberg, Acta Phys. Pol. 27, 3249 (1996); Phys. Lett. B 433, 236 (1998).

[17] B. Schenke and C. Greiner, Phys. Rev. C73, 034909 (2006).

[18] B. Mieck, H. Haug, W.A. Huegel, M.F. Heinrich and M. Wegener, Phys. Rev. B62, 2686 (2000); D.S. Chemla and J. Shah, Nature, 411, 549 (2001); V.M. Axt and T. Kuhn, Rep. Prog. Phys. 67, 433 (2004).

[19] S. Bosanas, Phys. Rev. A24, 777 (1981)

[20] P. Danielewicz and S. Pratt, Phys.Rev. C53, 249 (1996).

[21] J. Knoll and D.N. Voskresensky, Phys. Lett. B351, 43 (1995); Annals Phys. 249, 532 (1996).

[22] L. D. Landau and I. Pomeranchuk, Dokl. Akad. Nauk SSSR, 92 553, 735 (1953); A. B. Migdal, Phys. Rev. 103,1811 (1956); Sov. Phys. JETP 5, 527 (1957).

[23] P. L. Anthony, et al., Phys. Rev. Lett. 75, 1949 (1995).

[24] A.B. Migdal, E.E. Saperstein, M.A. Troitsky, and D.N. Voskresensky, Phys. Rep. 192, 179 (1990).

[25] J.D. Jackson, "Classical Electrodynamics." (Willey, N.Y., 1975), p. 302.

[26] V. Spicka, P. Lipavsky, and K. Morawetz, Phys. Lett. A240, 160 (1998); K. Morawetz, P. Lipavsky, and V. Spicka, Prog. Part. Nucl. Phys. 42, 147 (1999). 